\title{
Human Mesenchymal Stem Cells Promote Ischemic Repairment and Angiogenesis of Diabetic Foot Through Exosome miRNA-21-5p
}

\section{Chen Huang}

Guangzhou Panyu Central Hospital

\section{Wenfeng Luo}

Guangzhou Panyu Central Hospital

\section{Qian Wang}

Jinan University

Yufeng Ye

Guangzhou Panyu Central Hospital

\section{Jinghui Fan}

Guangzhou Panyu Central Hospital

Li Lin

Jinan University

\section{Congying Shi}

Institutionof Guangdong Cord Blood Bank

\section{Wei Wei}

Institution of Guangdong Cord Blood Bank

Hanwei Chen

Guangzhou Panyu Central Hospital

Yangzhe Wu ( $\nabla$ tyzwu@jnu.edu.cn )

Jinan University https://orcid.org/0000-0002-5270-2747

\section{Yukuan Tang}

Guangzhou Panyu Central Hospital

\section{Research}

Keywords: Diabetic foot, Exosomes, Mesenchymal Stem Cells, miRNA, Angiogenesis

Posted Date: July 29th, 2020

DOI: https://doi.org/10.21203/rs.3.rs-45894/v1 
License: (c) (i) This work is licensed under a Creative Commons Attribution 4.0 International License. Read Full License

Version of Record: A version of this preprint was published at Stem Cell Research on April 1st, 2021. See the published version at https://doi.org/10.1016/j.scr.2021.102235. 


\section{Abstract}

Background: Diabetic foot is caused by ischemic disease of lower extremities of diabetic patients, and the effective therapy is very limited. Mesenchymal stem cells (MSCs) based cell therapy had been developed into a new treatment strategy for diabetic foot clinically. However, the underlying molecular mechanism remains to be fully addressed. Exosomes secreted by MSCs may play crucial role in the processes of MSCs mediated inhibition of inflammatory microenvironment as well as pro-angiogenesis of ischemic tissue of diabetic foot.

Methods: Exosomes were isolated from MSCs using ultra-high centrifugation, and further characterized by the nanoparticle tracking analyzer and flow cytometry. Moreover, RNA sequencing, Western Blot, in vitro cell proliferation, in vivo pro-angiogenesis, as well as ischemic repairment of diabetic foot through rat model were performed to evaluate exosome physiological functions.

Results: We found that different inflammatory cytokines (tumor necrosis factor a, vascular cell adhesion molecule-1 and interleukin-6) induced MSCs to secrete exosomes heterogeneously, including exosome size and quantity. Through RNA sequencing, we defined a new proangiogenic miRNA, miRNA-21-5p. Further knockdown and overexpression of miRNA-21-5p by manipulating MSCs validated the biological activity of exosome miRNA-21-5p, including in vitro cell proliferation, in vivo pro-angiogenesis in Chick Chorioallantoic Membrane (CAM) assay, and in vivo pro-angiogenesis experiments (tissue injury and repair) in diabetic rat models. Furthermore, we discovered that exosome miRNA-21-5p promoted angiogenesis through upregulations of vascular endothelial growth factor receptor (VEGFR) as well as activations of serine/threonine kinase (AKT) and mitogen-activated protein kinase (MAPK). Together, our work suggested miRNA-21-5p could be a novel mechanism by which exosomes promote ischemic tissue repairing and angiogenesis. Meanwhile, miRNA-21-5p could be potentially developed into a new biomarker for exosomes of MSCs to treat diabetic foot.

Conclusions: miRNA-21-5p is a new biomarker and a novel mechanism by which exosomes promote ischemic tissue repairing and angiogenesis of diabetic foot. Our work could not only provide new scientific evidences for revealing pro-angiogenesis mechanism of MSCs, but also eventually benefit MSCs-based clinical therapy for diabetic foot of diabetes patients.

\section{Introduction}

During past decades, because of increasing aging population and changes in people's diets, the worldwide incidence of diabetes has increased rapidly. There has more than 420 million of diabetes patients worldwide according to WHO report. ${ }^{[1]}$ Diabetes can lead to various fatal diseases. For example, diabetic foot, caused by ischemic disease of lower extremities of diabetic patients, can lead to amputation and even death eventually. It has been reported that $15 \%$ of diabetic patients developed diabetic foot. ${ }^{[2]}$ The amputation rate of diabetic foot was about $25 \%-50 \%$, which is 40 times higher than 
that of non-diabetic population ${ }^{[3]}$. In addition, the 5 -year survival rate of the patients with diabetic foot was only about $50 \% .^{[3]}$

Diabetic foot is a type of complicated pathophysiological lesion related to a variety of factors. Most studies supported the hypothesis that diabetic foot is a kind of chronic, low-grade inflammatory disease $^{[4]}$. Various inflammatory factors, such as interleukin 6 (IL-6), tumor necrosis factor (TNF), and vascular endothelial cell adhesion molecule 1 (VCAM1), are involved in the development of diabetic foot. These inflammatory cytokines are significantly correlated with the development and prognosis of diabetic foot $^{[5,6]}$.

Despite advanced surgical and percutaneous transluminal angioplasty, treatment options for diabetic foot patients are still limited. Recently, mesenchymal stem cells (MSCs) based transplantation therapy had been shown to be able to improve perfusion and circulation of ischemia, including inducing vascular regeneration and reducing amputation rates of diabetic foot patients. ${ }^{[7]}$ MSCs-based therapy strategy has been played increasingly role in treatments of various types of diseases. As far as diabetic foot, MSCs could achieve ischemic repairment and angiogenesis ${ }^{[8]}$ of local impaired tissue, and thus exhibited sound clinical efficacy. However, it's controversial about the clinical long-term safety of MSCs, for example the concerning on the tumorigenesis possibility of MSCs. Therefore, development of new therapy methodology based on MSCs has been under continuous investigation.

Recent studies have reported that MSCs secrete exosomes, ${ }^{[9]}$ which are membranous vesicles with diameter of $30-150 \mathrm{~nm}$ and density of $1.10 \sim 1.18 \mathrm{~g} / \mathrm{ml} \cdot{ }^{[10]}$ Exosomes contain various molecules including proteins, mRNA, and microRNA (miRNA), and these molecules can regulate the gene network of target cells as novel intercellular communicators. ${ }^{[11-13]}$ During the past a few years, exosomes and exosome miRNA of MSCs in the angiogenesis had been increasingly attracted researcher's interests. Previous reports indicated that MSC exosome miRNAs had positive functions in the repair of diabetic foot. For instance, exosome miRNA-210-3p can promote angiogenesis and improve microcirculation by upregulating expression of VEGF gene and activating pro-angiogenic key proteins, such as SRC, AKT, ERK; [14] miRNA-146a could inhibit inflammatory factors and immune responses, thus promote diabetic tissue repair, ${ }^{[15]}$ miRNA-27b could inhibit apoptosis of diabetic endothelial progenitor cells; ${ }^{[16]}$ the Let-7 miRNA family could control the inflammatory response of diabetic atherosclerosis, ${ }^{[17]}$ miRNA-126 could promote ischemic tissue repair. ${ }^{[18]}$

Nevertheless, the underlying molecular mechanism on how exosomes promote angiogenesis in the inflammatory microenvironment remains largely unclear. For instance, it is yet to fully address whether inflammatory cytokines affect exosome secretion and components of MSCs in the context of inflammatory environment such as diabetic foot, and new functional exosome component molecules like miRNA remains to be further determined. Therefore, in this study, through in vitro and in vivo experimental models, we defined a new type of MSCs exosome, exosome miRNA-21-5p, and our results indicated that exosome miRNA-21-5p could potently promote vessel endothelial cell (HUVECs) proliferation and 
angiogenesis of diabetic foot. Altogether, our work will further our understandings on the underlying molecular mechanisms of MSCs in the therapy of ischemic injury induced diabetic foot.

\section{Methods}

\section{Mesenchymal stem cell culture}

Mesenchymal stem cells (MSCs) were provided by the Guangdong umbilical blood hematopoietic stem cell bank (Guangzhou, China). The phenotype of the MSCs was verified by flow cytometry, cell surface antigen markers including CD34, CD45, CD73, CD90, and CD105. MSCs were cultured in serum-free Dulbecco's Modified Essential Medium (DMEM)-F12 (HyClone, Logan, UT, USA) supplemented with either $2 \mathrm{mg} / \mathrm{ml}$ glucose (Control Group), $2 \mathrm{mg} / \mathrm{ml}$ glucose plus $20 \mathrm{ng} / \mathrm{ml}$ VCAM-1 (ADP5, R\&D, USA) (VCAM-1 Group), 2 mg/ml glucose plus 20 ng/ml TNF-a (T6674, Sigma, Germany) (TNF-a Group), or 2 mg/ml glucose plus 20 ng/ml IL-6 (200-06-20, PeproTech, USA) (IL-6 Group). The culture supernatant was collected after 48 hours of incubation.

\section{Isolation and purification of exosomes from MSCs}

Exosomes were isolated from the collected culture supernatant using ultracentrifugation by referring literatures ${ }^{[19]}$. Briefly, to remove cellular debris and proteins, the supernatant was sequentially centrifuged at $300 \mathrm{~g}$ for $5 \mathrm{~min}, 2000 \mathrm{~g}$ for $10 \mathrm{~min}$, and then $10000 \mathrm{~g}$ for $20 \mathrm{~min}$. The supernatant was then filtered using $0.22 \mu \mathrm{m}$ filter (UFC810096-1, Merck Millipore, Germany) to remove particles with over $0.22 \mu \mathrm{m}$ in size. The filtrate was then ultra-centrifuged at $100000 \mathrm{~g}$ for $120 \mathrm{~min}$ at $4^{\circ} \mathrm{C}$ (Optima XE-90 ultracentrifuge with a swing rotor, Type 70Ti; Beckman Coulter, CA, USA). The enriched MSCs-exo was resuspended in PBS for further studies.

\section{Morphological visualization using transmission electron microscopy (TEM)}

To analyze the morphological features of MSCs-exo, transmission electron microscopy (JEOL 2100F, Japan) was used here. For sample preparation, the MSCs-exo suspension was dropped onto the copper grid and deposited for $1 \mathrm{~min}$, following by treatment of $10 \mu \mathrm{l}$ uranyl acetate (phosphotungstic acid) for 1 min. Then, MSCs-exo sample stood by for $20 \mathrm{~min}$ in a dry environment, and then washed with $100 \mu \mathrm{l}$ PBS three times for 2 min before TEM observation.

\section{Nanoparticle tracking analysis (NTA)}

The MSCs-exo size and concentration were assessed using NanoSight LM10 system (Malvern). All the measurements were performed in triplicate at room temperature, with the detection threshold fixed to 20 - 
300 particles per field of view and the camera level set at standard mode for $1 \mathrm{~min}$. Data analyses were performed using NanoSight NTA software.

\section{Sequencing analysis of exosomal miRNA}

To reveal the miRNA components of exosomes, the MSCs-exo $\left(8 \times 10^{10}\right)$ was analyzed by BGISEQ-500 technology. Then the acquired data were compared with miRBase and other non-coding databases. We used Transcripts Per Kilobase Million (TPM) ${ }^{[20]}$ to normalize miRNA expression and predict target genes using RNAhybrid, ${ }^{[21]}$ miRanda ${ }^{[22]}$, and TargetScan ${ }^{[23]}$. Functional heat-maps were used to exhibit hierarchical clustering analysis of miRNA expressions. The hypergeometric test was then used to analyze significantly enriched gene ontology terms based on database (refers to http://www.geneontology.org/). Pathway-based analyses were used to discover the functional target genes using $\mathrm{KEGG}^{[24]}$ database.

\section{Knockdown(KD) and overexpression(OE) of miRNA-21-5p of MSCs}

According to miRNA sequencing results, we found significant high expression of miRNA-21-5p of exosomes secreted from all MSC groups, therefore, Knockdown(KD) and overexpression(OE) of miRNA21-5p of MSCs were conducted here. Briefly, the virus packaging system was a three-plasmid system: pspax2, pMD2G, and pCDH. The epithelioid cell line 293T was used as lentivirus packaging cells, and Escherichia coli strain DH5a was used as vectors of lentivirus and plasmid. The expression of miRNA-21$5 p$ was verified by qRT-PCR and the Takara kit.

\section{Confocal imaging}

To observe interactions between MSCs-exo and HUVECs, laser scanning confocal microscopy was used here. For fluorescence staining of exosomes, MSCs-exo $\left(8 \times 10^{9}\right)$, miRNA-21-5p-KD-exo $\left(8 \times 10^{9}\right)$, and miRNA-21-5p-OE-exo $\left(8 \times 10^{9}\right)$ were respectively incubated with green fluorescent dye PKH67 (MINI67-1KT, SIGMA) for 5 min at room temperature, followed by twice PBS wash using ultracentrifugation, as described previously ${ }^{10}$. Then, human umbilical vein endothelial cells (HUVECs) were co-cultured with either PBS (control), MSCs-exo, miRNA-21-5p-KD-exo or miRNA-21-5p-OE-exo for 4 hours at $37^{\circ} \mathrm{C}$ with $5 \%$ CO2. Subsequently, the cytoskeleton (tubulin-a) of Human umbilical vein endothelial cells (HUVECs) was stained with fluorescent mouse anti-human monoclonal antibody (1:100, CY-SC006, Cytoskeleton) according to the standard protocol provided by reagent producer Cytoskeleton Inc. Cell nucleus was stained with Hoechst ( $0.1 \mathrm{ul}$ added with $200 \mathrm{ul}$ ). The cells were washed twice with PBS between each step. Finally, cell samples were observed using laser scanning confocal microscopy (TCS SP8, Leica, Germany). 


\section{In vitro cellular proliferation assay}

To evaluate how MSCs-exo affects cell proliferation of HUVECs, $1 \times 10^{6}$ HUVECs were treated with PBS (control), MSCs-exo $\left(3 \times 10^{10}\right)$, miRNA-21-5p-KD-exo $\left(3 \times 10^{10}\right)$, or miRNA-21-5p-OE-exo $\left(3 \times 10^{10}\right)$ in serumfree medium for 24 hours. Then, cell proliferation of HUVECs was analyzed using the Ki67 assay kit and flow cytometry (FACsverse, BD corporation, America) by following standard protocol.

\section{In vitro wound-healing migration assay}

$1 \times 10^{6}$ HUVECs were in 6-well plate with serum-free medium. A scratching wound was manually generated by a sterile $200-\mu \mathrm{l}$ pipette tip. Pictures of the wound area were taken after $0,8,16$, and 24 hours of MSCs-exo treatments. The migration was analyzed using the ImageJ software.

\section{In vitro endothelial cell tube formation assay}

96-well culture plates were pre-coated with $50 \mu \mathrm{L}$ of growth-factor-reduced Matrigel (BD) (356234, Matrigel; Corning) at $37^{\circ} \mathrm{C}$ for 30 minutes. Then, $4 \times 10^{4}$ HUVECs cells per well were co-cultured with either PBS (control), or MSCs-exo $\left(5 \times 10^{9}\right)$, miRNA-21-5p-KD-exo $\left(5 \times 10^{9}\right)$, or miRNA-21-5p-OE-exo $\left(5 \times 10^{9}\right)$ with the pre-treated plates at $37^{\circ} \mathrm{C}$ in $5 \% \mathrm{CO}_{2}$ for 4 hours. Afterward, cells were visualized and tube formation was quantified. Tube number and branch points were statistically analyzed using ImageJ software. ${ }^{[14]}$

\section{Western Blot analysis}

To test expressions of functional proteins, Western Blot was used here by following the standard protocols of our lab ${ }^{[25]}$. HUVECs $\left(5 \times 10^{6}\right)$ were pre-treated with MSCs-exo $\left(5 \times 10^{9}\right)$ after 24 hours. VEGF, AKT, and MAPK signaling pathway molecules were then tested, including: VEGFR1 (1:1000 dilution, 2893S, Cell Signaling Technology, Danvers, MA, United), AKT (1:1000 dilution, 4691S, Cell Signaling Technology, Danvers, MA, United States), Phospho-AKT (1:1000 dilution, 13038S, Cell Signaling Technology, Danvers, MA, United States), ERK (1:2000 dilution, 4370S, Cell Signaling Technology, Danvers, MA, United States), and Phospho-ERK (1:1000 dilution, 4695S, Cell Signaling Technology, Danvers, MA, United States).

\section{Chorioallantoic membrane assay}

The pro-angiogenesis ability of MSCs-exo was examined using the chorioallantoic membrane (CAM) methodology. Fertilized chicken eggs (Gallus gallus) were rinsed and soaked in l:1000 benzalkonium bromide solution for three minutes. They were randomly divided into four groups and subsequently 
incubated at $37.8^{\circ} \mathrm{C}$. After seven days, a small opening was made in the shell. The chorioallantoic membrane was treated with either PBS (control), MSCs-exo $\left(5 \times 10^{9}\right)$, miRNA-21-5p-KD-exo $\left(5 \times 10^{9}\right)$, or miRNA-21-5p-OE-exo $\left(5 \times 10^{9}\right)$, and the picture were taken. The openings in the eggs were covered with adhesive tape and the eggs were returned to the incubator. After nine days, pictures were taken again to evaluate the pro-angiogenesis ability of the MSCs-exo.

\section{Hindlimb ischemia rat diabetic foot model}

9-week male Sprague-Dawley rats (300 - 350 g; grade: SPF; license \#: 1100111911009085) were purchased from Charles River Laboratory Animal Technology Co., Ltd. (Beijing, China; License: SCXK 2016-0011) to establish rat diabetic model. All animal experiments were conducted in accordance with the Institutional Animal Care and the Use Committee of Jinan University (License: SYXK 2017-0174). The Animal Experiment Protocol (Approval No.: IACUC-20190403-03) has been reviewed and approved by the Laboratory Animal Ethics Committee of Jinan University. Diabetes of rats was induced by intraperitoneally injected with $40 \mathrm{mg} / \mathrm{kg}$ (in $0.01 \mathrm{M}$ sodium citrate, $\mathrm{pH} 4.3$ ) streptozotocin. The blood glucose level of rats was controlled between 11.1 and $31.5 \mathrm{mmol} / \mathrm{L}$. Subsequently, the femoral artery and all arterial branches of rat diabetic model were ligated to establish diabetic foot model. In addition, $5 \mathrm{~mm}$ full-thickness skin of the dorsal hind foot of the diabetic rats was wounded to simulate diabetic injury model.

\section{In vivo treatment of diabetic foot rats using MSCs-exo}

According to RNA sequencing, we found miRNA-21-5p altered the most significantly. To address whether miRNA-21-5p played crucial role on the function of pro-angiogenesis of exosomes in the inflammatory microenvironment, we performed knockdown (KD) and overexpression (OE) of miRNA-21-5p in MSCs. The surface markers of the exosomes in all the groups were identified using flow cytometry.

Then, diabetic foot rats were randomly divided into four groups: control group (PBS), MSCs-exo group, miRNA-21-5p-KD-exo group, and miRNA-21-5p-OE-exo group. Rats from each group received intramuscular injections of $1 \mathrm{ml}$ PBS (control), MSCs-exo $\left(5 \times 10^{10}\right.$, mixed with $1 \mathrm{ml} \mathrm{PBS}$ ), miRNA-21-5pKD-exo $\left(5 \times 10^{10}\right.$, mixed with $\left.1 \mathrm{ml} \mathrm{PBS}\right)$, or miRNA-21-5p-OE-exo $\left(5 \times 10^{10}\right.$, mixed with $\left.1 \mathrm{ml} P B S\right)$ in the local area of ischemic hindlimb and foot ulceration. Afterward, we assessed the effect of MSCs-exo on proliferation signal molecules by measuring the expression of AKT and MAPK signaling pathway proteins by Western blot. The angiogenic VEGF signaling pathway proteins were measured by Western blot as well.

Additionally, the rat ischemic hindlimb severity degree of all groups including ischemic hindlimb complexion, skintemperature, and limb motor function were analyzed at D1, D7, and D14 after MSCs-exo treatments. The rat foot ulcer size of all rats was measured at D1, D3, and D7 after treatments, and then the ulcer area was analyzed using Image $\mathrm{J}$. 


\section{Ultrasonic Doppler perfusion imaging and contrast ultrasonography}

Ultrasonic Doppler imaging was performed to assess angiogenesis at D1 and D7 after treatment with MSCs-exo through the animal ultrasound micro imaging system (MYLAB 30CVVET, Esaote, Italy). Rats were anesthetized with $2 \%$ isoflurane, and ultrasound images of the right ischemic hindlimb were then immediately acquired using B mode Doppler perfusion imaging system. The blood perfusion ratio was analyzed by comparing the mean blood flow velocity and vessel number before and after treatments.

\section{Digital subtraction angiography}

Ischemic hindlimb angiogenesis was evaluated by Digital Subtraction Angiography (DSA) at D14 after treatment with MSCs-exo. The brief procedures include: isolation of femoral artery after incision of the rat's thigh skin, following by ioversol (H20067896, Hengrui, Jiangshu, China) injection by puncture with a tiny needle; subsequently, hindlimb vessels of the rat were observed by the digital subtraction angiography machine (INNOVA-3100-IQ, General Electric Company, USA). The vessel numbers of the pictures taken by DSA were analyzed by Image $\mathrm{J}$.

\section{Histology and immunohistochemistry}

At day 14 , muscle and ulceration tissue from the ischemic limb was harvested, Then, the tissues were fixed in $10 \%$ formalin, paraffin-embedded and sliced. The sliced tissues ( $3 \mu \mathrm{m}$ in slice thickness) were stained with hematoxylin and eosin (H\&E). To conduct immunohistochemical examinations of granulation tissue angiogenesis, rabbit polyclonal anti-CD31 antibody (1:300 dilution, GB12063, Servicebio, China), anti-VEGF antibody (1:100 dilution, GB11034, Servicebio, China), and anti-TGF $\beta$ antibody (1:1000 dilution, GB11271-1, Servicebio, China) were used for sample staining by following standard protocols.

\section{Statistical analysis}

All acquired research data were expressed as mean \pm standard deviation (SD). Significance between experimental groups and control group were calcuated using one-way ANOVA, and $p<0.05$ was considered statistically significant.

\section{Results}

\section{Exosome identification and effects of inflammatory cytokines on exosome size}


Firstly, to identify mesenchymal stem cells (MSCs), we used flow cytometry to characterize surface markers of MSCs. We found that the cultured cells express CD73, CD90, and CD105, which are mesenchymal stem cell markers, but not CD34 and CD45 that stand for hematopoietic cell markers (Fig. 1A). This verified that cells used for exosome isolation were MSCs. Subsequently, to validate exosomes, we applied flow cytometry to analyze surface markers of the MSC-exosomes of all groups, showing both CD63 and CD81 were highly expressed (Fig. 1B).

Due to the heterogeneity of individual MSCs, the molecular components of MSC-exosomes could be highly heterogenous in the context of inflammatory microenvironment. We thus selected three inflammatory cytokines (VCAM-1, TNF-a, IL-6) to test how the inflammatory factors affect exosomes from morphological aspects using TEM, and concentration of MSCs-exo was determined by NTA as well. It showed that exosomes of both control group and VCAM-1 treated group appeared to be sphere shape. As a contrast, exosomes from TNF-a or IL-6 stimulated groups appeared to be irregular shape (Fig. 1C). Nevertheless, exosome size showed similar distribution range, 30-350 nm with $154.1 \pm 42.2 \mathrm{~nm}$ in average diameter according to the NTA assay (Fig. 1D). Additionally, the exosome concentration (particle counts) was determined by NTA as well. According to calculation of projecting area of concentration curves, it showed that total exosome counts were $(7.42 \pm 0.51) \times 10^{8} / \mathrm{ml}$ (control group), $(1.10 \pm 0.16) \times$ $10^{9} / \mathrm{ml}$ (VCAM-1 group), $(7.37 \pm 0.31) \times 10^{8} / \mathrm{ml}$ (TNF-a group), and $(3.01 \pm 0.23) \times 10^{8} / \mathrm{ml}$ (IL-6 group) (Fig. 1D). These results revealed that the exosome concentration was different among four groups, which implicated with that exosome secretion could be regulated by different inflammatory cytokines.

Furthermore, to assess whether exosomes could be internalized by HUVECs, cells were incubated with fluorescent dye labeled exosomes and then observed by fluorescence microscopy in real-time. Representative images showed that exosomes entered HUVECs after 4 hours of co-incubation (Fig. 1E). Additionally, it's interesting to note that cytoskeleton tubulin of HUVECs was significantly upregulated, and distributed in a polarization manner.

\section{Sequencing analysis of exosome miRNA}

To further identify MSCs-exo from gene aspect, exosome miRNA sequencing was conducted. The MSCsexo of all groups were sequenced by BGISEQ-500 technology. Hierarchical cluster analysis of differentially expressed miRNA was exhibited as function heatmap (Fig. 2A), showing that miRNA-21-5p was the most expressed miRNA among inflammatory cytokines (VCAM1, TNFa, and IL-6) stimulated groups. Moreover, the expression level of miRNA-21-5p was calculated by using TPM (Transcripts Per Kilobase Million) from each group, which quantitatively showed that miRNA-21-5p was the most enriched miRNA of exosomes (Supp. Table). These results suggested that miRNA-21-5p would be a potent function player in the process of exosome mediated angiogenesis in the context of inflammatory microenvironment. 
Therefore, we focused our work on how exosomes would regulate angiogenesis through miRNA-21-5p. We conducted knockdown(KD) and overexpress(OE) of miRNA-21-5p expression in MSCs using the virus packaging system, and afterward the exosomes were isolated from MSCs supernatant. Through q-PCR assay of exosomes, we validated the methodology of knockdown (KD) and overexpression (OE) of miRNA-21-5p, showing KD could reduce miRNA-21-5p expression level, and OE could significantly elevate miRNA-21-5p expression (Fig. 2B).

\section{Exosomes promote endothelial cell proliferation and migration through miRNA-21-5p}

To study the role of exosomes in HUVECs proliferation, cell proliferation marker Ki67 was examined by flow cytometry (Fig. 3A). The results showed that exosome treatment could significantly promote Ki67 expression, and overexpression of miRNA-21-5p could more dramatically elevate Ki67 expression. As a contrast, knockdown of miRNA-21-5p exerted no statistical downregulation of Ki67 expression.

Moreover, we assessed cell migration ability in the absence and presence of exosomes. We thus performed the wound-healing assay, and results showed that overexpression of miRNA-21-5p could be more obvious to promote cell migration and wound healing, comparing with control, exosomes, and exosomes $^{\text {miRNA-21-5p-KD }}$ groups (Fig. 3B). Through statistical analyses, we found that exosomes ${ }^{\text {miRNA-21- }}$ $5 \mathrm{p}-\mathrm{OE}$ pre-treated HUVECs exhibited 2-fold faster cell migration and would healing rates compared to control group, and significant faster than exosomes ${ }^{\text {miRNA-21-5p-KD }}$ as well. Thus, such data clearly indicated that MSCs exosomes could promote proliferation and migration of HUVECs, and miRNA-21-5p played crucial role in these processes.

\section{miRNA-21-5p exosomes promote angiogenesis in vitro}

To further evaluate the angiogenic activity of exosome, HUVECs were treated with different types of exosomes, and then the formed endothelial cell tube and branches were visualized (Fig. 4A). We can clearly see that exosomes treatment could obviously promote the formation of endothelial tube. Importantly, miRNA-21-5p overexpressed exosomes had significantly higher activity to promote angiogenesis. Furthermore, statistical analyses quantitatively revealed that exosomes could significantly increase number of formed cell tubes. Specifically, normal exosomes elevated the number of cell tubes by 2-fold comparing to the control group, and exosomes ${ }^{\text {miRNA-21-5p-OE }}$ increased the tube number by 4-fold, but exosomes ${ }^{\text {miRNA-21-5p-KD }}$ reversely reduced such increases (Fig. 4B). Additionally, similar results were obtained by counting the branching points per tube (Fig. 4C). It clearly showed exosomes ${ }^{\text {miRNA-21-5p-OE }}$ significantly increased the branching points by 4 -fold comparing with the control group, whereas normal exosomes only increased about 2-fold. Such data again indicated that miRNA-21-5p played important role in regulating angiogenesis in the context of exosome and HUVECs interaction. 


\section{miRNA-21-5p exosomes promote HUVEC proliferation through AKT and MAPK phosphorylation}

Since exosomes could promote HUVEC proliferation, cell migration and in vitro angiogenesis, we thus further detected expressions of key proteins related to cell proliferation using Western Blot (WB). As shown in Figure 5, total expression level of AKT and MAPK as well as phosphorylated AKT and MAPK were examined (A). Meanwhile, vascular endothelial growth factor receptor 1 (VEGFR1) was also detected by the WB (Fig. 5B). According to quantitative analyses, we could see that p-AKT and p-MAPK expression levels of HUVECs were significantly elevated in the context of exosome ${ }^{\text {miRNA-21-5p-OE }}$ treatments. As a contrast, exosomes ${ }^{\text {miRNA-21-5p-KD }}$ could dramatically reduce $\mathrm{p}$-AKT expression, but only had minor effect on p-MAPK (Fig. 5C). As for VEGFR1 expression, it unequivocally showed that exosomes could significantly upregulate VEGFR1 expression, and exosome miRNA-21-5p-OE further elevated this upregulation. These results altogether indicated that exosomes could promote the proliferation of HUVECs by activating AKT and MAPK as well as upregulating VEGFR1, and that miRNA-21-5p played crucial role in the processes of functional regulation.

\section{miRNA-21-5p exosomes accelerate angiogenesis in vivo}

To evaluate the in vivo angiogenesis ability of exosomes, the Chick Chorioallantoic Membrane (CAM) assay was conducted, and representative imaging results were shown as Figure 6A. Further, we quantified number of the formed blood vessels of four groups, and the statistical result was shown in Figure 6B. It revealed that exosomes could significantly promote the generation of blood vessels, and miRNA-21-5p-OE further promoted angiogenesis, but miRNA-21-5p-KD could attenuate such promotion.

\section{miRNA-21-5p exosomes promote ulceration repair of diabetic foot in hindlimb ischemia model of rats}

To further investigate the angiogenesis effect of miRNA-21-5p exosomes in vivo, we established diabetic foot model by ligating femoral artery of diabetic rats. Afterward, ischemic limbs of rats were treated with PBS, exosomes, exosomes ${ }^{\text {miRNA-21-5p-KD }}$, or exosomes ${ }^{\text {miRNA-21-5p-OE }}$, and pictures with or without exosome treatments were then taken at day 0, 7 and 14, as shown in Figures 7 (A-D). Meanwhile, mean repair area, ischemic hindlimb complexion scores, skin temperature scores, and limb motor function scores were statistically analyzed as well (Figs. 7E-7H). From pictures, we could see that exosome treatment could visually speed up ulceration repair of diabetic foot, and exosomes ${ }^{\text {miRNA-21-5p-OE }}$ exhibited the most optimal repairing capability. Further statistical data (repair area and scores) also apparently indicated that treatments of exosomes, particularly exosomes ${ }^{\text {miRNA-21-5p-OE }}$, significantly improved foot ulcer healing in diabetic rats (Figs. 7E-7H). 


\section{miRNA-21-5p exosomes promote the ischemic hindlimb blood perfusion in vivo}

Here, we further studied the angiogenesis ability of exosomes from the aspect of blood perfusion. We used ultrasound imaging to visualize in vivo blood perfusion at day 1 and day 7 . The images in Figure 8 (A-D) showed that exosomes significantly enhanced the blood perfusion in ischemic hindlimb. Moreover, statistical results further indicated that exosomes, particularly exosomes ${ }^{\text {miRNA-21-5p-OE }}$, strikingly enhanced blood perfusion ratio (Fig. 8E).

At the meantime, the ischemic hindlimb angiogenesis was also analyzed by the Digital Subtraction Angiography (DSA) at day 14 post exosome treatment (Fig. 9A). We calculated the number of blood vessels visualized by images, and found that exosomes significantly elevated the formed blood vessels. Further statistical data clearly verified that exosomes ${ }^{\text {miRNA-21-5p-OE }}$ could enhance angiogenesis by about 3-fold compared to the control group. Nevertheless, miRNA-21-5p-KD depressed exosome potentiation on angiogenesis (Fig. 9B).

Additionally, we further examined ischemic hindlimb tissue using histochemical H\&E methodology 14 days post ischemia and treatment with exosomes. The imaging results revealed an increase of new formed vessel of exosome treated groups, and exosomes ${ }^{\text {miRNA-21-5p-OE }}$ group induced such increase the most significantly (Supp. Fig. A). At the meanwhile, immunohistochemistry showed the blood vessel density of the ischemic hindlimb tissue (CD31, TGF $\beta$, or VEGF staining) was apparently enhanced after treatment with exosomes (Supp. Fig. B), which was further confirmed by statistical analyses (Supp. Fig. C). It clearly indicated that the blood vessel density of exosomes ${ }^{\text {miRNA-21-5p-OE }}$ group was the highest, followed by exosome group and then exosomes ${ }^{\text {miRNA-21-5p-KD }}$ group. Together, tissue staining suggested that miRNA-21-5p played a key role in angiogenesis and tissue repair in vivo.

\section{Discussion}

During the past decades, diabetes has emerged as one of major chronic diseases impairing health and live quality. Diabetic foot, as one of disabling and even fatal diseases, is basically caused by ischemic legs of the diabetes patients. In the past a few years, infusion of mesenchymal stem cells (MSCs) had become a new therapy strategy clinically to control or cure diabetic foot. However, the fundamental mechanism on how MSCs repair the ulceration and promote angiogenesis of local impaired tissue of diabetic foot remains to be fully addressed. Previous literatures generally considered that the multidirectional differentiation potential of MSCs plays the major role in tissue repair ${ }^{[26]}$. Nevertheless, exosome secreted by MSCs has now been proposed to play crucial role in the process of tissue repair as well ${ }^{[27]}$.

Exosomes could regulate target cells through binding to receptors on the cell surface or phagocytosis by cells directly, and thus act as intercellular signal transducers. ${ }^{[28]} \mathrm{MSC}$ exosomes can stimulate 
endothelial cells to generate blood vessels and upregulate angiogenesis related genes. ${ }^{[29]}$ The angiogenesis mechanism involves in various miRNAs of exosomes, which are non-coding microRNAs comprising approximately 22 nucleotides. ${ }^{[30]}$ Given exosome contains various functional proteins and miRNA molecules, further investigation on exosome function and discovering new exosome miRNA will provide us new scientific evidences for fully revealing the underlying molecular mechanism of tissue repair and pro-angiogenesis of inflammatory diabetic foot of MSCs therapeutics.

The occurrence of diabetic foot is primarily due to impaired angiogenesis in a high-glucose and inflammatory environment. ${ }^{[18]}$ Inflammatory cytokines such as TNF- $\alpha$ and IL-6 were associated with poor prognosis. In the present work, we firstly evaluated how the inflammatory cytokine that normally exists in the inflammatory microenvironment of diabetic foot would affect exosomes secreting by MSCs. We selected thee type of inflammatory factors, TNF-a, VCAM- 1 and IL-6, to test the potential effects on exosomes, and found that inflammatory factors (TNF-a, VCAM-1 and IL-6) affected not only the morphology and quantity of the MSCs exosomes, but also the miRNA components of exosomes. It revealed that VCAM- 1 could stimulate exosome production by MSCs the most significantly, following by TNF-a and IL-6. Further sequencing analyses of exosome miRNA suggested that miRNA-21-5p was the most enriched miRNAs. To identify the biological function of miRNA-21-5p in the tissue repair and angiogenesis of diabetic foot, we thus obtained exosomes ${ }^{\text {miRNA-21-5p-KD }}$ and exosomes ${ }^{\text {miRNA-21-5p-OE }}$ by manipulating MSCs.

Through Ki67 expression and wound-healing experiments, we confirmed that miRNA-21-5p played crucial role in promoting the proliferation and migration of HUVECs in vitro. Moreover, in vitro cell tubule formation assay also revealed that exosomes ${ }^{\text {miRNA-21-5p-OE }}$ treatments produced significantly higher numbers of tubular and branches, implicating with stronger capability of angiogenesis. Further molecular results indicated that exosomes, particularly exosomes ${ }^{\text {miRNA-21-5p-OE }}$, could facilitate phosphorylation of AKT and MAPK, which are crucial regulation proteins of cell activation and proliferation, as well as expression of VEGFR1 that is one of key receptors relating with angiogenesis.

Further in vivo experiments including Chick Chorioallantoic Membrane (CAM) assay and rat model confirmed exosomes had promising pro-angiogenesis activity, including greater number of generated blood vessel, faster wound healing of ischemic area, and improved blood perfusion ratio. Notably, the exosomes $^{\text {miRNA-21-5p-OE }}$ had significant higher activity in the CAM model and the diabetic foot ischemic model of rats. Therefore, our present work revealed new insights into the angiogenesis mechanisms of MSCs exosomes, and suggested that miRNA-21-5p upregulated exosomes had greater profound benefit for inhibiting the diabetic inflammatory environment than normal exosomes. Together, our in vitro and in vivo results unequivocally indicated that miRNA-21-5p was vital for pro-angiogenesis of exosomes. Importantly, miRNA-21-5p could be potentially developed into a new biomarker for exosomes of MSCs to treat diabetic foot. The whole mechanism of MSC exosomes promote proliferation and migration of HUVECs as well as angiogenesis through miRNA-21-5p could be sketchily illustrated as Fig. 10. 


\section{Conclusions}

In this work, we discovered that exosome miRNA-21-5p promotes angiogenesis through upregulations of VEGFR as well as activations of AKT and MAPK signaling pathway. Unlike MSCs, which can further proliferate and differentiate in vivo and thus have concerns on possibility of tumorigenesis, exosomes possess better clinical safety. Because exosome ${ }^{\text {miRNA-21-5p }}$ could also exert sound therapeutic efficacy similar as MSCs, exosome miRNA-21-5p will be a promising alternative for diabetic foot therapy in addition to MSCs. Moreover, our work suggested miRNA-21-5p could be a novel regulation mechanism by which exosomes promote ischemic tissue repairing and angiogenesis, and it could be potentially developed into a new biomarker for exosomes of MSCs to treat diabetic foot. Our further work will focus on how exosome miRNA-21-5p would inhibit inflammatory responses of diabetic foot, specifically the antiinflammatory mechanisms from aspects of immune cells including CD4+, CD8 + T cells, NK cells and macrophages. Altogether, this work could not only provide new scientific evidences for revealing proangiogenesis mechanism of MSCs, but also eventually benefit MSCs-based clinical therapy for diabetic foot of diabetes patients.

\section{List Of Abbreviations}

MSCs, mesenchymal stem cells; WB, western blot; TNF- $\alpha$, tumor necrosis factor $\alpha$; VCAM-1, vascular endothelial cell adhesion molecule 1; IL-6, interleukin-6; CAM, chick chorioallantoic membrane; AKT, serine/threonine kinase; MAPK, mitogen-activated protein kinase; VEGFR1, vascular endothelial growth factor receptor 1; miRNA, micro ribonucleic acid (RNA); KD, knockdown; $O E$, overexpression; $C D$, cluster of differentiation; NTA, nanoparticle tracking analyzer; TEM, transmission electron microscopy.

\section{Declarations:}

\section{Ethical Approval and Consent to participate}

The Animal Experiment Protocol has been approved by the Laboratory Animal Ethics Committee (Approval No.: IACUC-20190403-03) and the Institutional Animal Care and the Use Committee (License: SYXK 2017-0174) of Jinan University.

\section{Consent for publication}

Not applicable.

\section{Availability of data and material}

Not applicable.

\section{Competing interests}

The authors declare no competing interests 


\section{Funding}

This work was supported by research funding from Guangzhou Health Science and Technology Project (Grant Number:20191A011120) and Panyu Science and Technology Plan Medical General Project (Grant Number: 2018-Z04-47).

\section{Author contributions}

C. Huang: project design, experiment performing, data analyzing and manuscript drafting. Y. Wu: project design, experiment instruction, data analyzing, research resources providing, manuscript writing, revision, and proof-reading. Q. Wang, J. Fan, W. Luo, Y. Ye, L. Lin, C. Shi, and W. Wei: data analyses and discussion. $\mathrm{H}$. Chen and Y. Tang: experiments design, data analyses and discussion. All the authors approved the final manuscript.

\section{Acknowledgments}

We gratefully acknowledge the Institution of GuangDong Cord Blood Bank for providing the MSCs. We also thank Jiawei Li, Fengyi Mai, Yi Hu, Xiaoyan Lin, Huijie Xing and others from the Biomedical Translational Research Institute of Jinan University for help and suggestions on experiments.

\section{References}

1. Mendis S, Davis S, Norrving B. Organizational Update: The World Health Organization Global Status Report on Noncommunicable Diseases 2014; One More Landmark Step in the Combat Against Stroke and Vascular Disease. Stroke. 2015;46:e121-e2.

2. Xu Y, Wang L, He J, Bi Y, Li M, Wang T, et al. Prevalence and Control of Diabetes in Chinese Adults. JAMA. 2013;310:948-59.

3. Wu Q, Chen B, Liang Z. Mesenchymal Stem Cells as a Prospective Therapy for the Diabetic Foot. Stem cells International. 2016;2016:4612167.

4. Shi E, Jiang X, Wang L, Akuzawa S, Nakajima Y, Kazui T. Intrathecal Injection of Hepatocyte Growth Factor Gene-modified Marrow Stromal Cells Attenuates Neurologic Injury Induced by Transient Spinal Cord Ischemia in Rabbits. Anesthesiology. 2010;113:1109-17.

5. Rubtsov Y, Goryunov K, Romanov A, Suzdaltseva Y, Sharonov G, Tkachuk V. Molecular Mechanisms of Immunomodulation Properties of Mesenchymal Stromal Cells: A New Insight into the Role of ICAM-1. Stem Cells International. 2017;2017:1-15.

6. Zaka M, Abbasi BH, Durdagi S. Novel tumor necrosis factor-a (TNF-a) inhibitors from small molecule library screening for their therapeutic activity profiles against rheumatoid arthritis using target-driven approaches and binary QSAR models. Journal of Biomolecular Structure and Dynamics. 2019;37:2464-76.

7. Chen Y, yu B, Xue G, Zhao J, Li R-K, Liu Z, et al. Effects of Storage Solutions on the Viability of Human Umbilical Cord Mesenchymal Stem Cells for Transplantation. Cell transplantation. 
2013;22:1075-86.

8. Mendelson A, Frenette PS. Hematopoietic stem cell niche maintenance during homeostasis and regeneration. Nature Medicine. 2014;20:833-46.

9. Baglio S, Rooijers K, Koppers-Lalic D, Verweij F, Lanzón M, Zini N, et al. Human bone marrow- and adipose-mesenchymal stem cells secrete exosomes enriched in distinctive miRNA and tRNA species. Stem cell research \& therapy. 2015;6:127.

10. Komaki M, Numata Y, Morioka C, Honda I, Tooi M, Yokoyama N, et al. Exosomes of human placentaderived mesenchymal stem cells stimulate angiogenesis. Stem Cell Research \& Therapy. 2017;8:219.

11. Phinney D, Di Giuseppe M, Njah J, Sala E, Shiva S, Croix C, et al. Mesenchymal stem cells use extracellular vesicles to outsource mitophagy and shuttle microRNAs. Nature Communications. 2015;6:8472.

12. Kourembanas S. Exosomes: Vehicles of Intercellular Signaling, Biomarkers, and Vectors of Cell Therapy. Annual review of physiology. 2015;77:13-27.

13. Li P, Kaslan M, Lee S, Tan J, Gao Z. Progress in Exosome Isolation Techniques. Theranostics. 2017;7:789-804.

14. Gangadaran P, Rajendran R, Lee HW, Senthilkumar K, Hong CM, Jeong S, et al. Extracellular vesicles from mesenchymal stem cells activates VEGF receptors and accelerates recovery of hindlimb ischemia. Journal of Controlled Release. 2017;264:112-26.

15. Xu J, Wu W, Zhang L, Dorset-Martin W, Morris M, Mitchell M, et al. The Role of MicroRNA-146a in the Pathogenesis of the Diabetic Wound-Healing Impairment. Diabetes. 2012;61:2906-12.

16. Li H, Liu J, Wang Y, Fu Z, Huttemann M, Monks T, et al. MiR-27b augments bone marrow progenitor cell survival via suppressing the mitochondrial apoptotic pathway in Type 2 diabetes. American Journal of Physiology - Endocrinology And Metabolism. 2017;313:E391-E401.

17. Brennan E, Wang B, McClelland A, Mohan M, Marai M, Beuscart O, et al. Protective Effect of let-7 miRNA Family in Regulating Inflammation in Diabetes-Associated Atherosclerosis. Diabetes. 2017;66:2266-77.

18. Mathiyalagan P, Liang Y, Kim D, Misener S, Thorne T, Kamide C, et al. Angiogenic Mechanisms of Human CD34(+) Stem Cell Exosomes in the Repair of Ischemic Hindlimb. Circulation research. 2017;120:1466-76.

19. Tooi M, Komaki M, Morioka C, Honda I, Iwasaki K, Yokoyama N, et al. Placenta Mesenchymal Stem Cell Derived Exosomes Confer Plasticity on Fibroblasts. Journal of Cellular Biochemistry. 2016;117:1658-70.

20. t Hoen PAC, Ariyurek Y, Thygesen HH, Vreugdenhil E, Vossen RHAM, de Menezes RX, et al. Deep sequencing-based expression analysis shows major advances in robustness, resolution and inter-lab portability over five microarray platforms. Nucleic Acids Research. 2008;36:e141.

21. Krüger J, Rehmsmeier M. RNAhybrid: microRNA target prediction easy, fast and flexible. Nucleic Acids Research. 2006;34:W451-W4. 
22. John B, Enright AJ, Aravin A, Tuschl T, Sander C, Marks DS. Human MicroRNA Targets. PLOS Biology. 2004;2:e363.

23. Agarwal V, Bell GW, Nam J-W, Bartel DP. Predicting effective microRNA target sites in mammalian mRNAs. eLife. 2015;4:e05005.

24. Benjamini Y, Yekutieli D. The Control of the False Discovery Rate in Multiple Testing under Dependency. The Annals of Statistics. 2001;29:1165-88.

25. Hu Y, Liu T, Li J, Mai F, Li J, Chen Y, et al. Selenium nanoparticles as new strategy to potentiate $\gamma \delta T$ cell anti-tumor cytotoxicity through upregulation of tubulin-a acetylation. Biomaterials. 2019;222:119397.

26. Sayed N, Liu C, Wu JC. Translation of Human-Induced Pluripotent Stem Cells. Journal of the American College of Cardiology. 2016;67:2161-76.

27. György B, Szabó TG, Pásztói M, Pál Z, Misják P, Aradi B, et al. Membrane vesicles, current state-ofthe-art: emerging role of extracellular vesicles. Cellular and Molecular Life Sciences. 2011;68:266788.

28. Thuma F, Zöller M. Outsmart tumor exosomes to steal the cancer initiating cell its niche. Seminars in Cancer Biology. 2014;28:39-50.

29. Ha M, Kim VN. Regulation of microRNA biogenesis. Nature Reviews Molecular Cell Biology. 2014;15:509-24.

30. Condorelli G, Latronico MVG, Cavarretta E. microRNAs in Cardiovascular Diseases. Journal of the American College of Cardiology. 2014;63:2177-87.

\section{Figures}




\section{Figure 1}

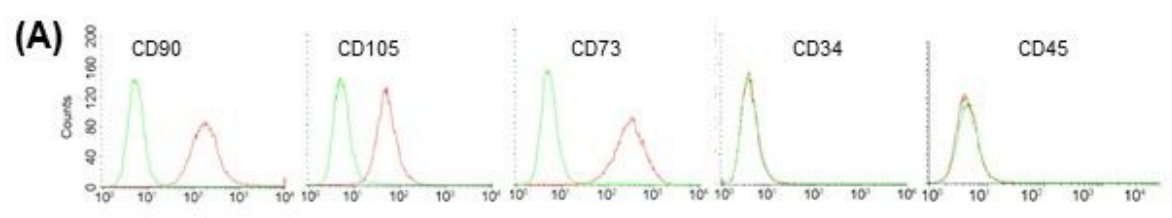

(B)
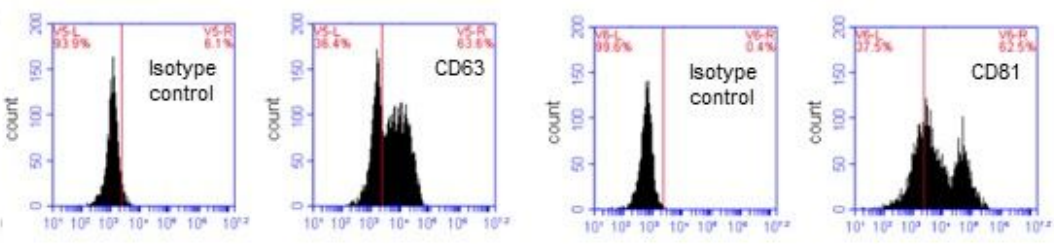

(C)

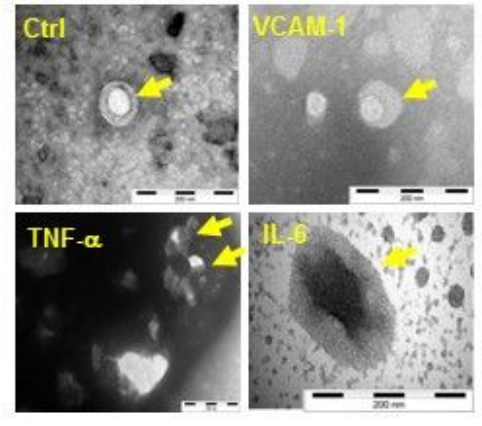

(D)

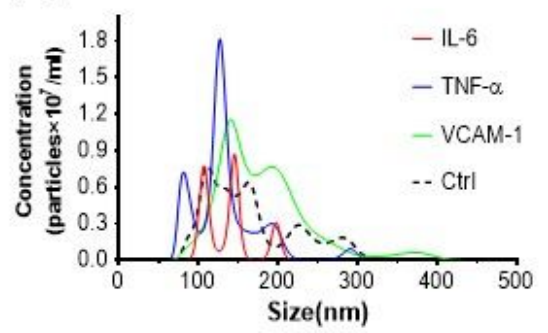

(E)
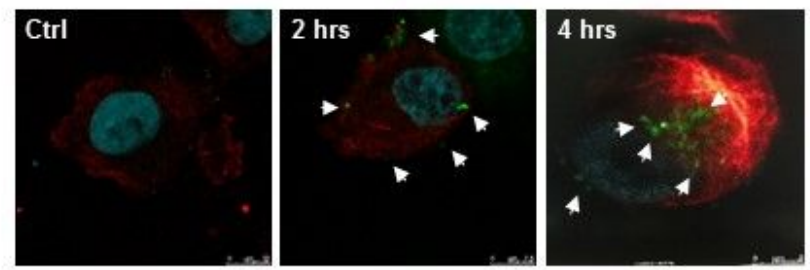

Tubulin: red

Nucleus: blue

Exosomes: green

\section{Figure 1}

Identification of human mesenchymal stem cells (MSCs) and exosomes. (A) Analysis of MSC-related cell surface markers using flow cytometry, showing that CD90 is $99.67 \%$, CD105 is $98.36 \%$, CD73 is $99.70 \%$, as a contrast, CD34 is only $0.1 \%$, and CD 45 is only $0.08 \%$ as well. (B) Flow cytometry identification of exosome through surface markers, CD63 (63.6\%) and CD81 (62.5\%). (C) Electron microscopic images of MSCs exosomes (MSC-exo) (scale bar, $200 \mathrm{~nm}$ ). Control group, exosome (yellow arrow) isolated from 
MSCs without any treatment; experimental group, exosomes (yellow arrow) isolated from VCAM-1, TNF-a, or IL-6 pretreated MSCs. (D) MSC-exo size distribution determined by NTA, showing exosome size distribution and exosome concentration (number per $\mathrm{ml}$ ) range. (E) Confocal visualization of interaction between HUVECs and exosomes (green particles pointed by white arrows).

\section{Figure 2}

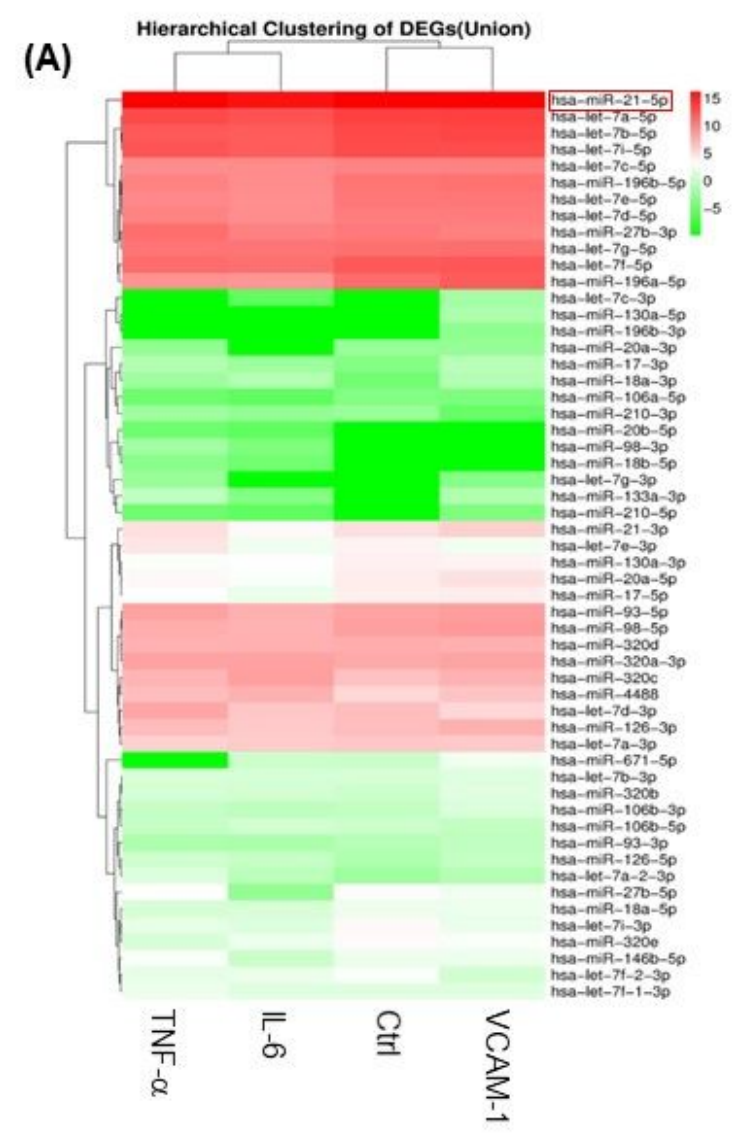

(B)

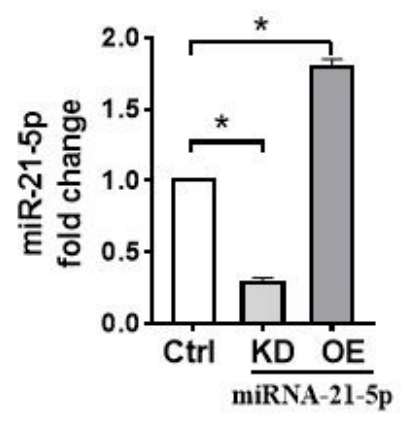

Figure 2 
Sequencing analysis of MSCs exosomes (MSC-exo). (A) (B) Heat map of miRNA expression of exosomes, which were isolated from MSCs that pre-treated with inflammatory cytokines including VCAM-1, IL-6, or

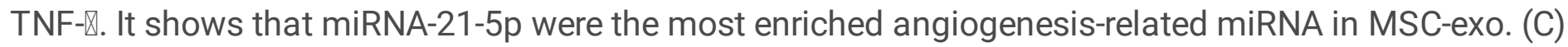
qPCR validation of miRNA-21-5p expression in exosomes, which were isolated from MSCs with miRNA21-5p knockdown (KD) or overexpression (OE). * $\mathrm{P}<0.05, \mathrm{n}=3$.

\section{Figure 3}
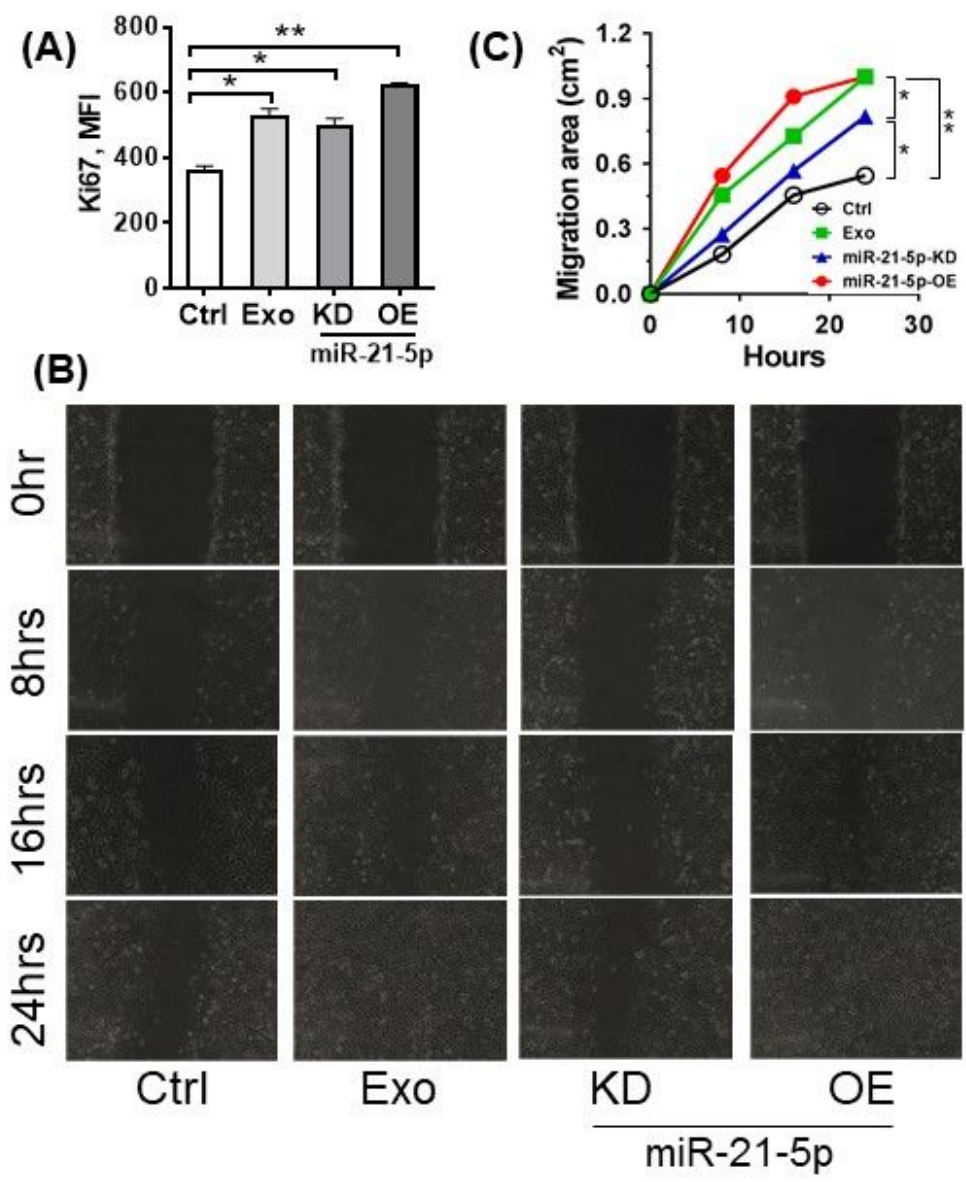

Figure 3 
Exosomes promote endothelial cell proliferation and cell migration of HUVECs in vitro. (A) Flow cytometry analysis of HUVEC Ki67 expression in the absence and presence of MSC exosomes (24 hours of interaction; miRNA-21-5p-KD, and miRNA-21-5p-OE). ${ }^{*}<<0.05$, $* * P<0.01$. (B) HUVEC wound \& healing observation using phase contrast microscopy. HUVECs were treated without or with exosomes (miRNA21-5p-KD or miRNA-21-5p-OE), and treatment time was $0,8,16$, and 24 hours. Images were acquired using 10x objective. (C) Quantitative analyses of cell migration by evaluating change rate of woundhealing area. ${ }^{*} \mathrm{P}<0.05,{ }^{*} \mathrm{P}<0.01$.

\section{Figure 4}

\section{(A)}
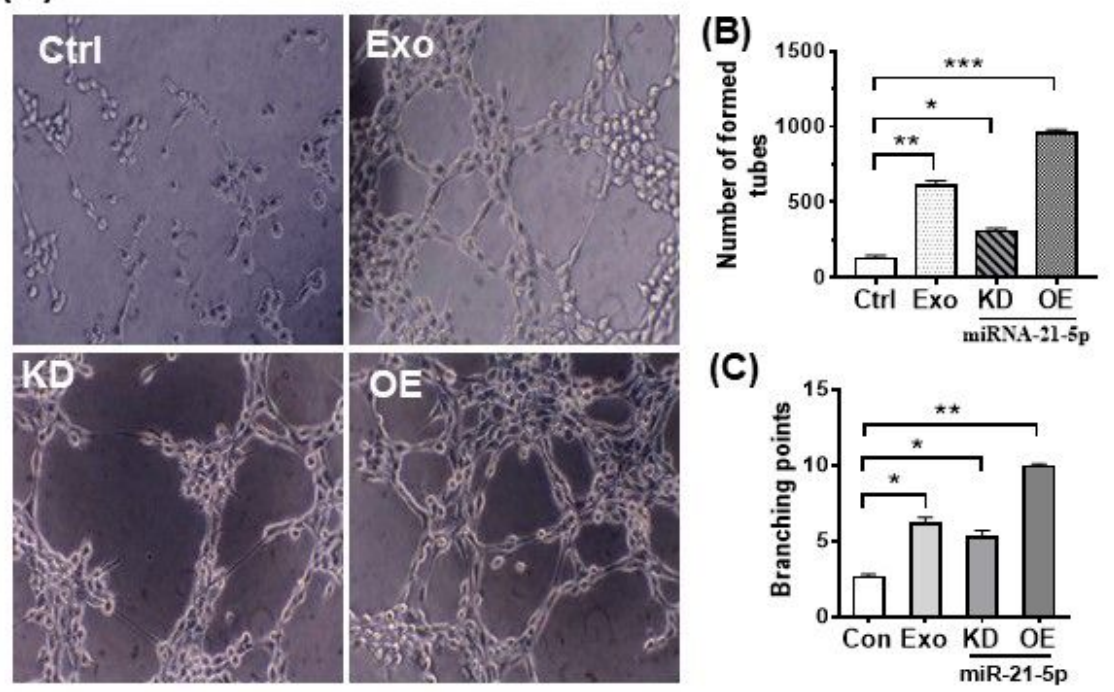

(C)

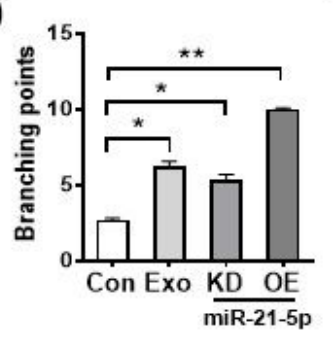


Figure 4

In vitro angiogenesis experiment by observing endothelial cell tube formation in the absence and presence of exosomes. (A) Optical microscopy images of tube formation of HUVECs pre-treated without or with exosomes (naive exosome, exosomemiRNA-21-5p-KD, exosomemiRNA-21-5p-OE) for 4 hours. 20x objective was used here. (B, C) Quantitative analyses of tubule formation by counting formed tubes and branching points, which were statistically analyzed from 6 different fields for each sample of triplicate experiments. ${ }^{*} \mathrm{P}<0.05,{ }^{*} \mathrm{P}<0.01$.

\section{Figure 5}

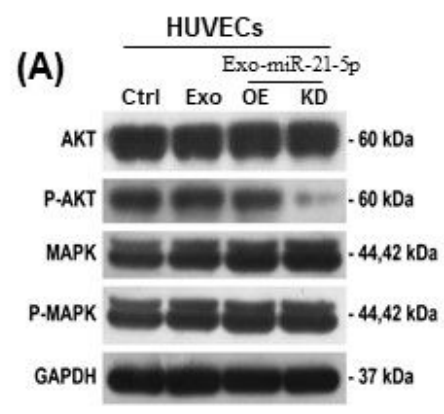

(C)
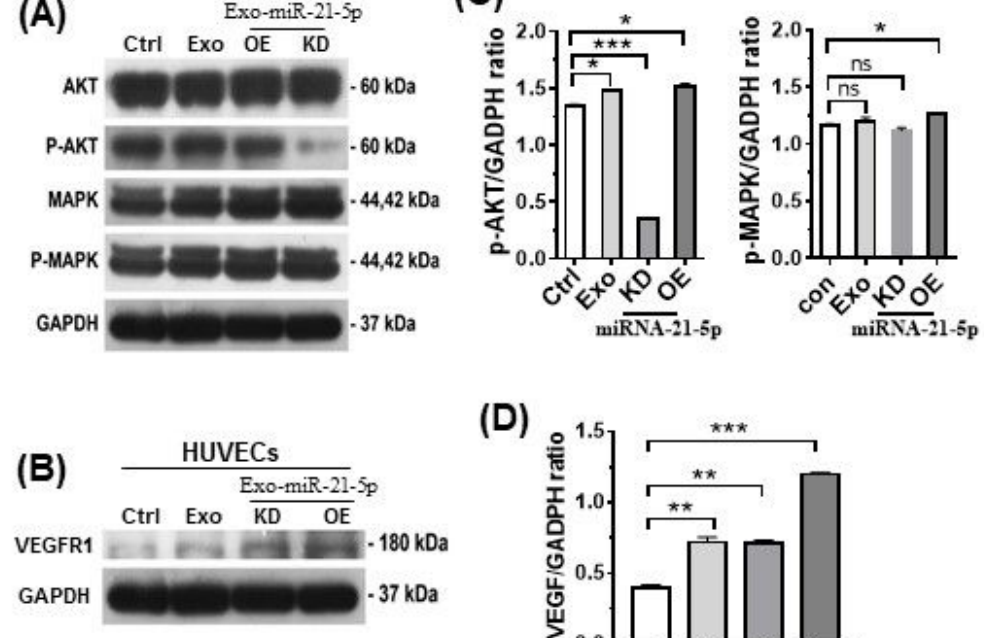

(D)

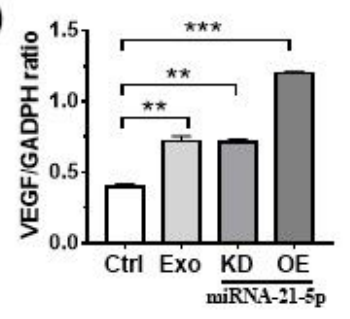


Figure 5

Exosome promote HUVEC proliferation through phosphating AKT. (A) Expressions of total AKT and MAPK as well as phosphorylated AKT and MAPK analyzed by Western Blot. HUVECs were pretreated with PBS (Ctrl), MSCs-exo, exosomemiRNA-21-5p-KD (KD), or exosomemiRNA-21-5p-OE (OE). (B) VEGF1 expression of HUVECs in the absence and presence of exosomes. (C, D) Quantitative comparisons of expressions of p-AKT, p-MAPK, VEGF1 among groups. ${ }^{*} P<0.05$, ${ }^{\star *} \mathrm{P}<0.01$, ${ }^{\star \star \star} \mathrm{P}<0.001$.

\section{Figure 6}

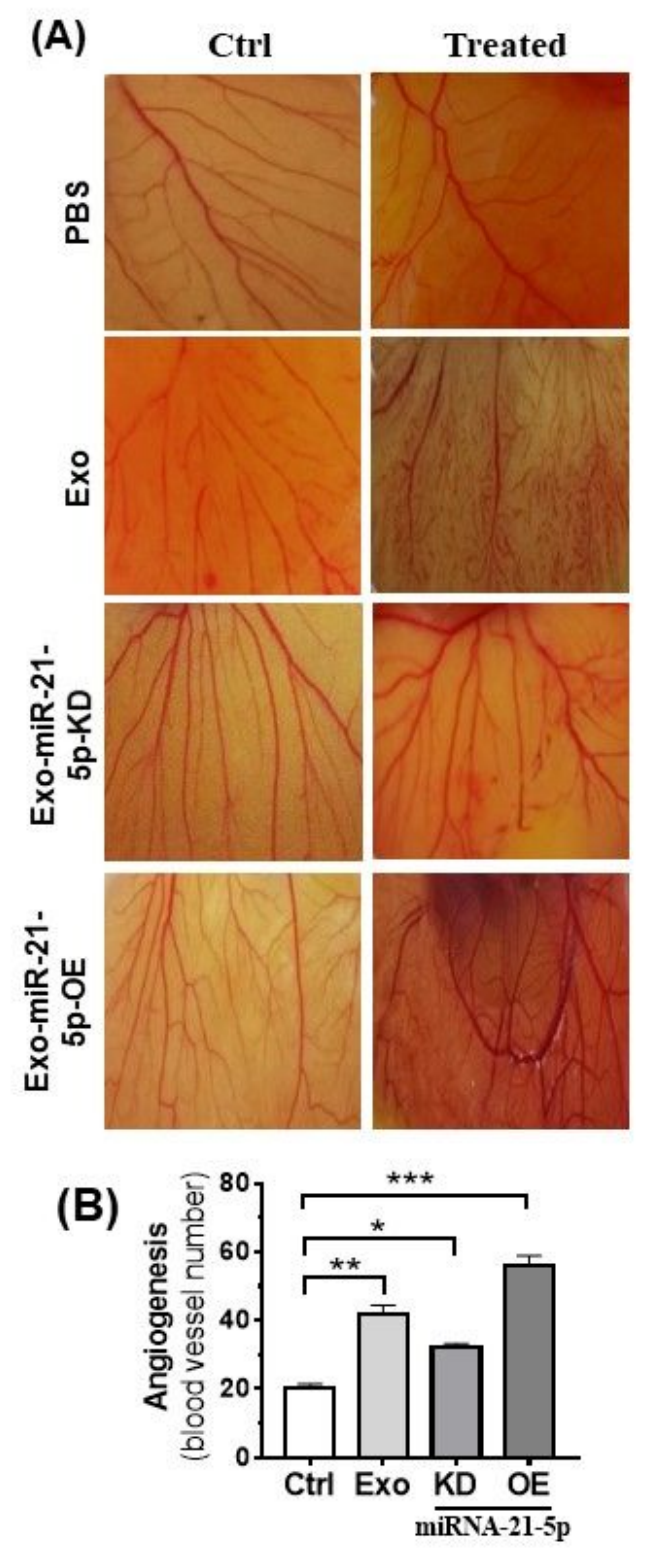


Figure 6

Exosome promoting in vivo angiogenesis validated by the Chick Chorioallantoic Membrane (CAM) assay. (A) Pictures from 4 groups, including PBS group, exosome group, miRNA-21-5p-KD exosome group, and miRNA-21-5p-OE exosome group. (B) Statistical analyses of angiogenesis, showing exosomes could promote angiogenesis in vivo. ${ }^{*} \mathrm{P}<0.05, \star \star \mathrm{P}<0.01, \star \star \star \mathrm{P}<0.001$.

\section{Figure 7}

(A)
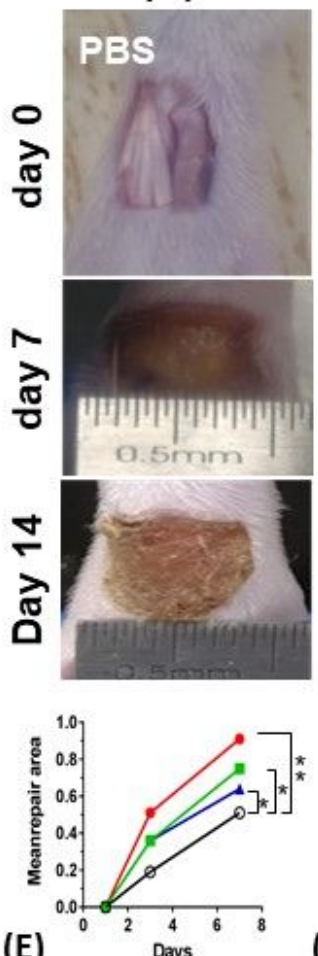

(E)

(B)
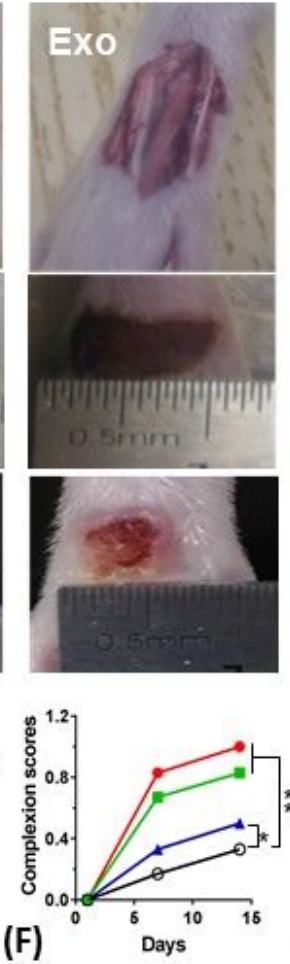

(C)

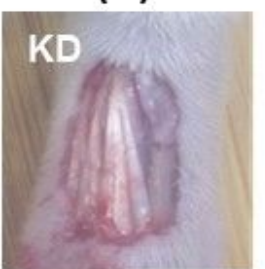

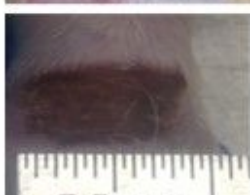
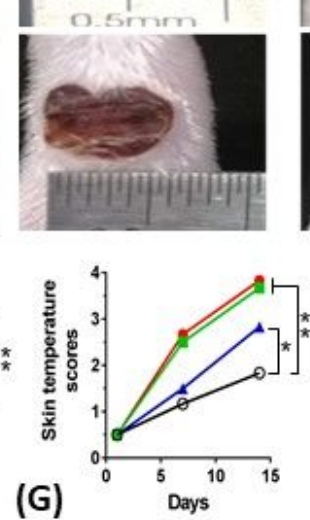

(D)
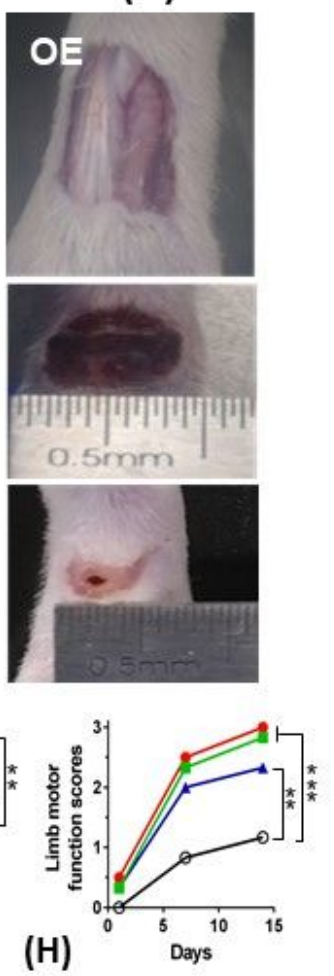

$\theta$ Ctrl $\neq$ miR-21-5p-KD

- Exo $\rightarrow$ miR-21-5p-OE

Figure 7 
Exosomes promote ischemic repair and angiogenesis of diabetic foot in rat model experiments. (A-D) Representative pictures of full-thickness skin defects of diabetic rats before and after treatments with exosomes. Pictures were taken at day 0 , day 7 , and day 14 post-operation. Concentration of exosomes used for treatments was $5 \times 1010$ in 1 ml PBS. Exo, naïve exosomes; KD, exosomemiRNA-21-5p-KD; OE, exosomemiRNA-21-5p-OE. (E) Quantitative analyses of mean repair area of diabetic foot ulceration in rats, data collected at day 1,3 , and 7 after treatment. Mean repair area $=$ (Beginning area-Measured area)/Beginning area. (F) Complexion scores of the ischemic limb (0 - pale, 1 - ruddy). (G) Temperature scores of the ischemic limb (on a scale of 0-4; 0 - Obvious cold without improvement after insulation, 1 Obvious cold with improvement after insulation, 2 - cool, 3 - slightly cool, and 4 - normal). (H) Limb motor function scores of the ischemic limb (on a scale of $0-3 ; 0$ - poor and 3 - strong). ${ }^{*} P<0.05,{ }^{\star} * P<0.01$, $\star \star \star P<0.001$. 


\section{Figure 8}
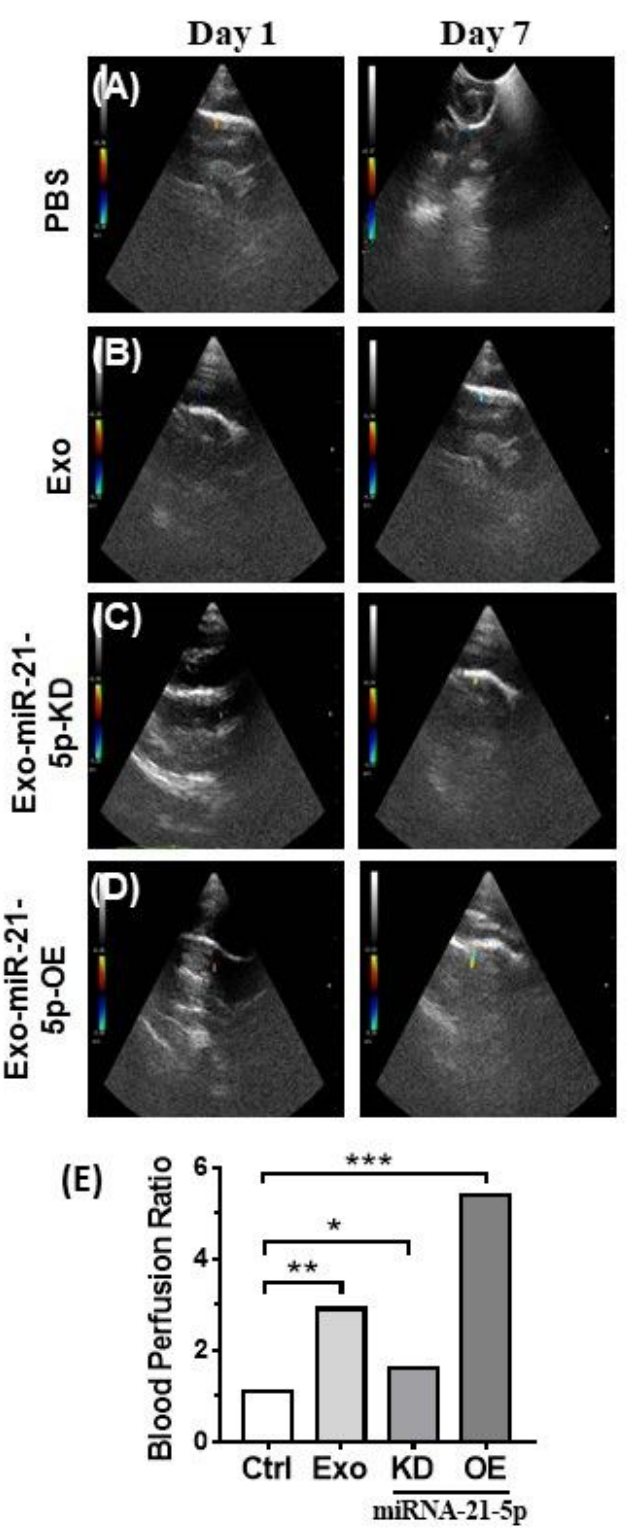

\section{Figure 8}

Blood perfusion in the ischemic hindlimb of rats visualized by ultrasound Doppler perfusion imaging. (AD) Representative images of blood perfusion after treatments with PBS, exosome, exosomemiRNA-21-5pKD, or exosomemiRNA-21-5p-OE. Images were acquired at day 1 or day 7. (E) Statistical analyses of blood perfusion ratio. Blood Perfusion Ratio $=($ mean blood flow velocity after treatment $\times$ vessel number 
after treatment $) /\left(\right.$ mean blood flow velocity before treatment $\times$ vessel number before treatment). ${ }^{*}<0.05$, ${ }^{*} \mathrm{P}<0.01, * \star * \mathrm{P}<0.001$.

\section{Figure 9}
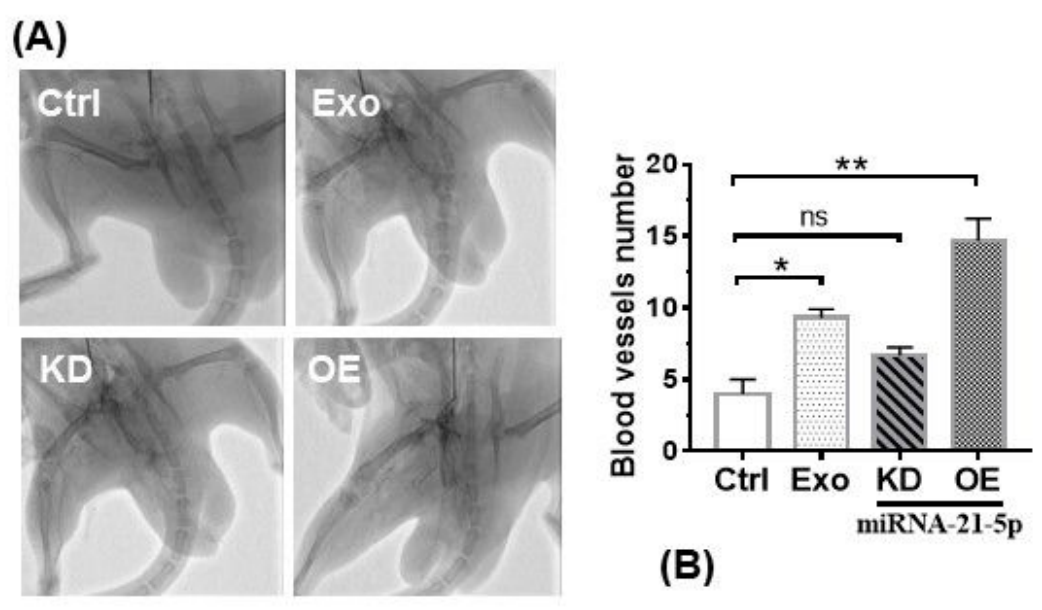

(B)

\section{Figure 9}

The ischemic hindlimb angiogenesis was analyzed by the Digital Subtraction Angiography (DSA) at day 14 post exosome treatment. $P<0.05$. (A) Representative images of ischemic hindlimb angiogenesis of rats in the absence (ctrl) and presence of exosomes. KD, exosomemiRNA-21-5p-KD; OE, exosomemiRNA- 
21-5p-OE. (B) Statistical comparisons of angiogenesis of the ischemic limb. ns, no significance, ${ }^{*} \mathrm{P}<0.05$ $\star \star P<0.01$.

\section{Figure 10}

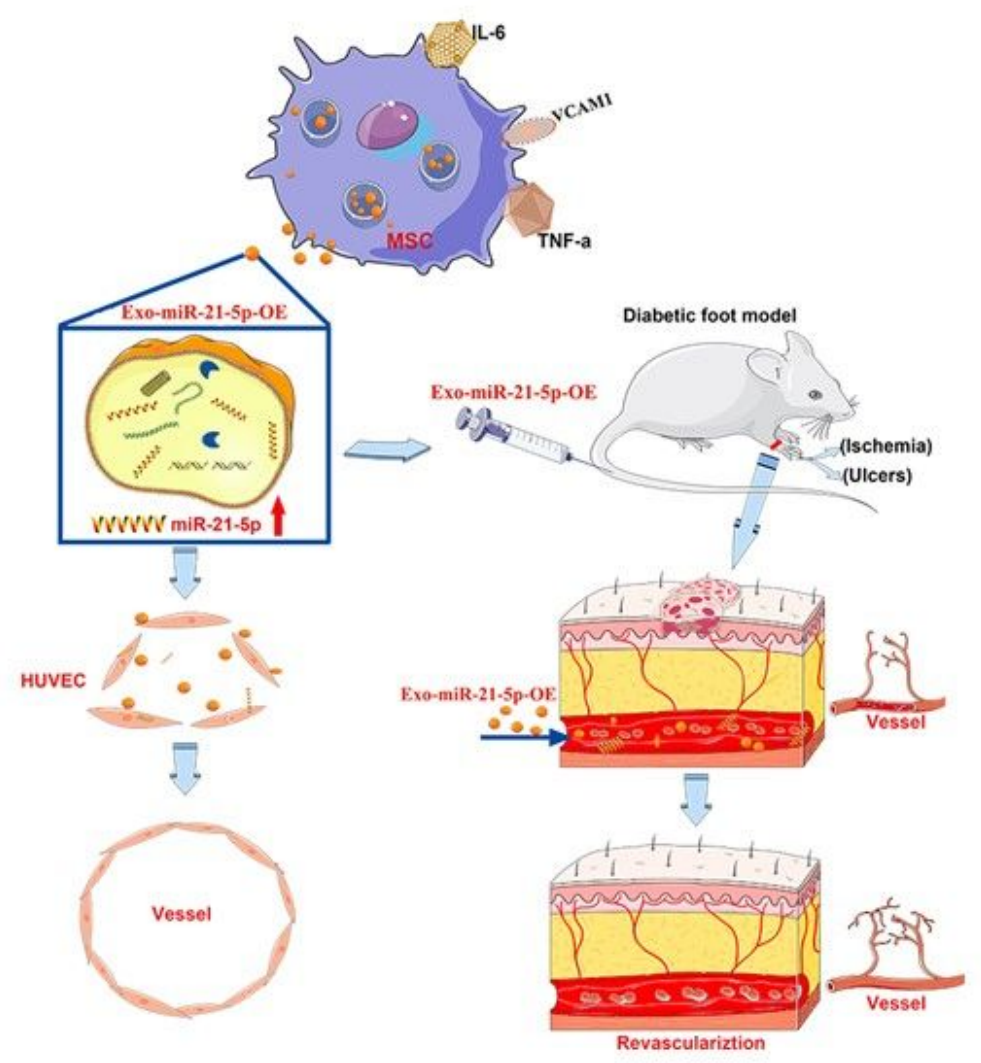

Figure 10

Sketch diagram shows that mesenchymal stem cells could secret exosomes, which could promote HUVEC proliferation and angiogenesis, and thus achieved ischemic repairment and angiogenesis of diabetic foot. During these processes, the miRNA-21-5p of exosomes played crucial roles. 


\section{Supplementary Files}

This is a list of supplementary files associated with this preprint. Click to download.

- 3Suppl.Material.pptx 